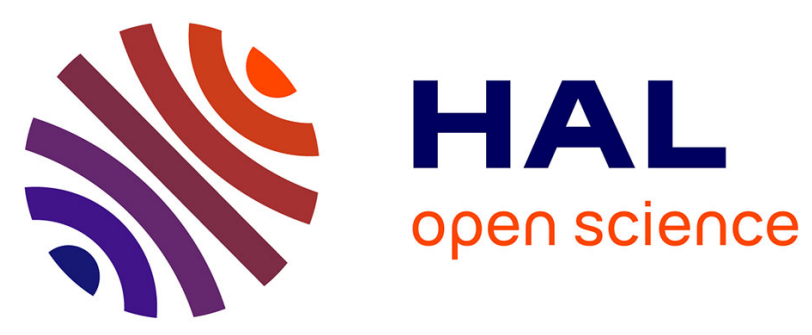

\title{
AUTOMATED SEGMENTATION OF RETINAL LAYERS IN OCT IMAGING AND DERIVED OPHTHALMIC MEASURES
}

Florence Rossant, Itebeddine Ghorbel, Isabelle Bloch, Michel Paques, Sarah Tick

\section{To cite this version:}

Florence Rossant, Itebeddine Ghorbel, Isabelle Bloch, Michel Paques, Sarah Tick. AUTOMATED SEGMENTATION OF RETINAL LAYERS IN OCT IMAGING AND DERIVED OPHTHALMIC MEASURES. ISBI, Jun 2009, Boston, United States. 10.1109/ISBI.2009.5193320 · hal-02882945

\section{HAL Id: hal-02882945 \\ https://hal.science/hal-02882945}

Submitted on 28 Jun 2020

HAL is a multi-disciplinary open access archive for the deposit and dissemination of scientific research documents, whether they are published or not. The documents may come from teaching and research institutions in France or abroad, or from public or private research centers.
L'archive ouverte pluridisciplinaire HAL, est destinée au dépôt et à la diffusion de documents scientifiques de niveau recherche, publiés ou non, émanant des établissements d'enseignement et de recherche français ou étrangers, des laboratoires publics ou privés. 


\title{
AUTOMATED SEGMENTATION OF RETINAL LAYERS IN OCT IMAGING AND DERIVED OPHTHALMIC MEASURES
}

\author{
Florence Rossant ${ }^{1}$, Itebeddine Ghorbel ${ }^{1,2,3}$, Isabelle Bloch ${ }^{2}$, Michel Paques ${ }^{4}$, Sarah Tick ${ }^{4}$ \\ ${ }^{1}$ ISEP Paris, ${ }^{2}$ Télécom ParisTech CNRS UMR 5141 LTCI Paris, ${ }^{3}$ FOVEA Pharmaceuticals Paris \\ ${ }^{4}$ Clinical Investigation Center 503, Centre Hospitalier National des Quinze-Vingts Paris. \\ Florence.Rossant@isep.fr, Itebeddine.Ghorbel|Isabelle.Bloch@enst.fr, Michel.Paques|Sarah.Tick@gmail.com
}

\begin{abstract}
This paper proposes an automated method for the segmentation of eight retinal layers in high resolution OCT images. It has been evaluated based on comparison with manual segmentation performed by five different experts. The method has been successfully applied on a database of 72 images. Quantitative measures are then derived as an aid to ophthalmic diagnosis. A good agreement with measures derived from manual segmentation is obtained which allows us to use the proposed method for retinal variability studies.
\end{abstract}

Index Terms- OCT retinal imaging, Automated segmentation, Quantitative evaluation

\section{INTRODUCTION}

Recently, optical coherence tomography (OCT) has revolutionized the clinical imaging of the retina, the photosensitive tissue of the eye. The spectral domain OCT technology now reaches a 5-10 $\mu \mathrm{m}$ resolution while the high acquisition speed reduces motion artifacts, allowing reliable image averaging [1]. These new technologies have contributed to improve the OCT image quality, and innovative segmentation approaches can now be proposed, for reaching a better accuracy and a better reliability. Then, measures derived from the segmented retinal layers (Fig.1) will help to better understand the anatomy of the human fovea.

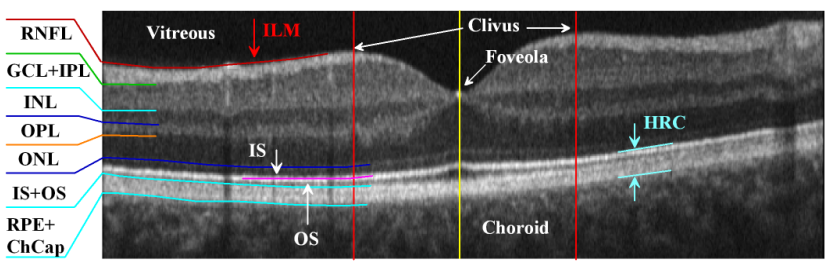

Fig. 1. Cross-sectional OCT image of the retina, acquired with the 3D OCT-1000, Topcon [1], and retinal layer definition.

Several retina segmentation methods have already been presented in the literature. They all start with a pre-processing step that allows reducing the speckle noise: mean filter [2], median filter [3,4], directional filters [5] or diffusion filters [6,7]. Then, most authors make extensive use of local boundary detection methods, as local profile analysis or local gradient analysis [2-6], which are not very robust to noise and varying contrast, and lack global consistency. Consequently, tests are required to select and label the significant edges: continuity check $[2,3,6,7]$, comparison with retina models $[3,5]$, thus limiting the practical use of these techniques in a clinical environment. These approaches require a lot of parameters, empirically tuned (e.g. [2,7]) or set through a large training set [3] which is again a limitation for clinical routine. Global optimizations are sometimes used to refine the first segmentation result $[4,7]$. But in all cases, not all retinal layers are detected (from two [3] to six $[5,6]$ ), and the segmentation is not always made around the foveola [6,7].

To overcome these limits, we propose a new method, based on more global segmentation algorithms, such as active contours, k-means and Random Markov Fields. Another originality of our approach is the modeling of the approximate parallelism between layers, based on a Kalman filter. As a result, eight retinal layers can be determined, including the inner and outer segments of the photoreceptors, which, to our knowledge, was not yet addressed in existing methods.

The remainder of the article is organized as follows: the segmentation method is presented in Section 2, experiments, including quantitative measurements, are discussed in Section 3.

\section{SEGMENTATION}

The images were acquired with the 3D OCT-1000, Topcon [1], to provide vertical and horizontal retina cross-sections of $6 \mathrm{~mm}$ length. The images were stored with two resolutions. We use the knowledge we have about the relative position of the retinal layers and how they appear in the OCT images, to detect and localize sequentially the retinal layers. Figure 2 presents the main steps that will be described in the next sub-sections:

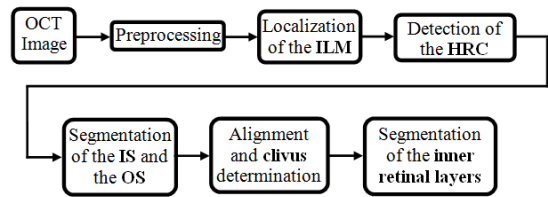

Fig. 2. Flowchart of the methodology.

\subsection{Preprocessing}

A non linear diffusion filter [8] is applied to the image (Fig. 3). This filter performs better than median or Gaussian filters since edges are better preserved. The output image is then normalized between 0 and 1 . In what follows, we denote by $W$ (typically 600 or 780 pixels, depending on the image resolution) and $H$ the image width and height. The coordinates are defined by the origin at the top left corner, the vertical $\mathrm{x}$-axis and the horizontal $\mathrm{y}$-axis. 


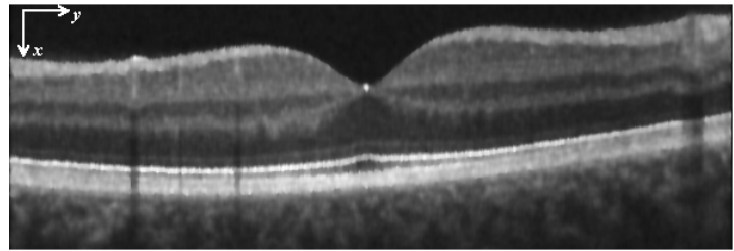

Fig. 3. Pre-processed image.

\subsection{Localization of the inner limiting membrane (ILM)}

The contrast between the inner retina and the vitreous is high enough to detect the ILM by an edge-tracking algorithm, based on the maximization of the local mean gradient. The result is then refined and regularized by applying an active contour algorithm. The energy functional is defined by internal forces, the rigidity and the tension of the contour, and external forces, the GVF field derived from the gradient image [9].

Then, the foveola $F\left(x_{F}, y_{F}\right)$ is defined as the ILM curve pixel whose $\mathrm{x}$-coordinate is maximal in the central area (Fig. 4).

\subsection{Detection of the hyper-reflective complex (HRC)}

This area, including the ChCap, RPE and OS layers, appears as a high intensity image strip above the choroid (Fig. 4). A Gaussian 1-D filter is applied column-wise, to provide a vertically smoothed image $S(x, y)$. The maximum response below $x_{F}$ corresponds to an inner pixel of the searched area. Starting from this point, the HRC median line is iteratively deduced, column by column, by looking for the maximum output of the following recursive low-pass filter:

$$
C(x, y)=(1-\alpha) S(x, y)+\alpha C(x, y \pm 1)
$$

(- from left to right, + from right to left)

In this method, the $S(x, y)$ coefficients are continuously integrated before making the decision, in order to model the continuity of the searched layer. Setting $\alpha=0.75$ leads to a regular median line, despite the residual noise and the retinal blood vessel shades (Fig. 4).

The cumulative profile computed along the median line, centered on it, allows us to determine the approximate distance between the median line and the HRC edges. Thus, it is possible to initialize an active contour [9], which will converge accurately towards the boundaries (Fig. 4). This result will be refined in the next steps to separate the RPE + ChCap layer from the OS layer.

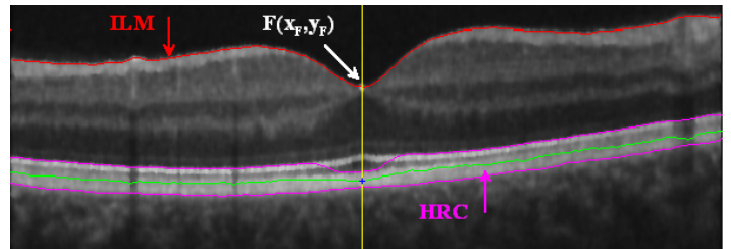

Fig. 4. Detection of the ILM and the HRC. In green, the median line of the HRC detected by recursive low-pass filtering.

\subsection{Segmentation of the photoreceptor segments (IS, OS)}

The junction between the inner (IS) and the outer (OS) segments of the photoreceptors appears as a bright narrow band just above the $\mathrm{RPE}+\mathrm{ChCap}$ layer, with a larger separation around the foveola $\mathrm{y}-$ coordinate. We apply a peak detector on an image area determined with respect to HRC. The maxima are detected in each column and labeled in order to form peak lines (Fig. 5a). The nearby peak lines are then merged, based on proximity criteria, and the gaps are filled using a linear interpolation (Fig. 5b). Then, the IS/OS junction is extracted by selecting the peak line that minimizes the distance to the inner side of the hyper-reflective complex.
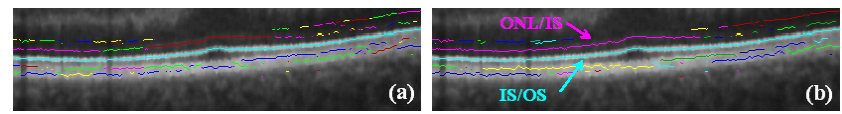

Fig. 5. Detection of the IS/OS junction by peak intensity analysis: peak lines before (a) and after (b) concatenation.

The interface between the ONL and the IS layers corresponds to a second fine line, parallel to the IS/OS junction, but less bright and more noisy. We propose to apply a Kalman filter [10] to track column by column the ONL/IS boundary. The Kalman filter is defined by a state vector $X$, that characterizes the tracked pixels, and a theoretical model that allows predicting the evolution of $X$. In our application, the state vector includes the intensity value of the pixel and its distance to the IS/OS line, and both values are supposed to be constant along the searched curve. Note that the distance provides an information about the parallelism between lines, as an original feature of our approach. State noise and measurement noise variances complete the model. All the parameters are dynamically initialized from the longest peak line found just above the IS/OS junction, except the state noise which has been set for all images. The Kalman equations formalize the principle of prediction/verification, realized in 3 steps. Firstly, the state vector $\hat{X}(n \mid n-1)$ at the column $n$ is predicted from the previous estimate $\hat{X}(n-1 \mid n-1)$, according to the evolution model. Secondly, a measure is made in the image, by seeking the maximum intensity pixel around the predicted position. Thirdly, the error between the prediction and the measure is computed to correct the prediction and to provide the new state vector estimate $\hat{X}(n \mid n)$. The correction is weighted by the filter gain, which is also adjusted at each iteration. Nevertheless, when no image pixel fits the prediction, the predicted value is kept and no measure is integrated in the filter. This allows overcoming the problem of short interruptions. The process stops after several iterations without acceptable measures. The proposed method leads to an accurate localization of the ONL/IS boundary, despite the low contrast and the noise, as illustrated in Figure 6.

The foveola coordinates are then refined by minimizing the distance between the ILM and the IS/OS junction.

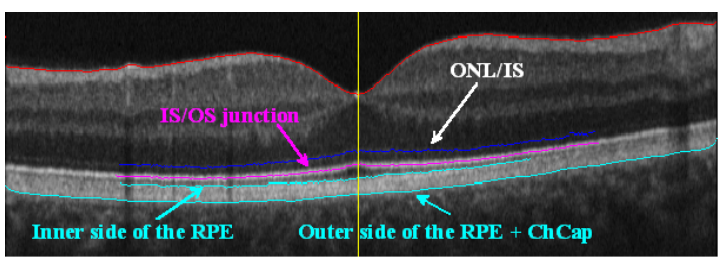

Fig. 6. ONL/IS and OS/RPE edges detected by Kalman filtering.

A second Kalman filter is applied on the gradient image, to deduce the inner side of the RPE layer from the outer side of the RPE + ChCap layer, since both curves are almost parallel (Fig. 6).

This method leads to very good results, even when retinal blood vessel shades cross the layers, but fails for some blurred images, without any contrast between the RPE and the OS layers. 


\subsection{Alignment and clivus determination}

The image is aligned against the outer side of the RPE + ChCap layer. The clivus is defined by the two highest points of the ILM found on both sides of the foveola, denoted by $\left(x_{C l}, y_{C D}\right)$ and $\left(x_{C r}, y_{C r}\right)$, as illustrated in Figure 7:

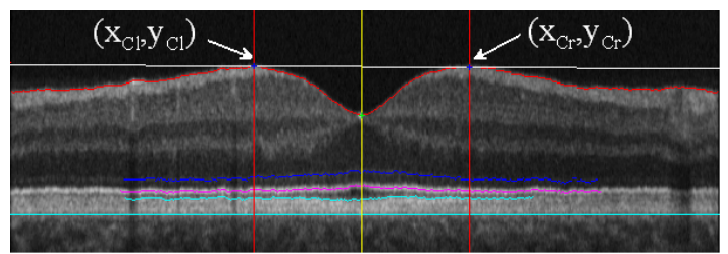

Fig. 7. Alignment and clivus determination.

\subsection{Inner layer segmentation (RNFL, ONL, GCL+IPL, INL)}

The inner retinal layers are segmented using Markov random field (MRF) based techniques [11]. The Markovian hypothesis allows us to take into account spatial interactions between connected pixels. In our application, the classification is performed according to the Bayesian maximum a posteriori (MAP) criterion, i.e. by looking for the label configuration that maximizes the probability of the class field (the labels) conditionally to the observation field (the intensity image). This optimal configuration corresponds to a minimum state of an energy function, defined as follows.

Let us denote by $P\left(f_{S} \mid \omega_{S}=i\right)$ the probability distribution of the pixel intensities, conditional to the class $i$. Based on a statistical analysis of the inner retinal regions, we proved that the image noise is Gaussian. The probability distribution is then defined by two parameters, the mean $\mu_{i}$ and the standard deviation $\sigma_{i}$. We use the Potts model $\varphi\left(\omega_{s}, \omega_{t}\right)$ to express the interactions between the 8connected sites $s$ and $t$. Under these assumptions, the energy function is defined by:

$$
U(\omega \mid f)=\sum_{s}\left(\frac{\left(f_{s}-\mu_{\omega_{s}}\right)^{2}}{2 \sigma_{\omega_{s}}{ }^{2}}+\ln \left(\sqrt{2 \pi} \sigma_{\omega_{s}}\right)\right)+\beta \sum_{(s, t)} \varphi\left(\omega_{s}, \omega_{t}\right)
$$

The first term is related to the image data, while the second one is a regularization term. The parameter $\beta$, empirically set, weights the relative influence of both.

We first apply the k-means algorithm to initialize the labels and estimate automatically the noise parameters $\left(\mu_{i}, \sigma_{i}\right)$ from the classification results. So, the segmentation process is fully unsupervised. Then, the energy function is minimized by running the simulated annealing (SA) algorithm. This segmentation method is applied several times, on the non pre-processed aligned image, the region of interest being adjusted each time. The contours are deduced from the labeled image, and regularized by a snake algorithm [9]. More details are given in what follows for each layer. The final results are shown in Figure 11 of Section 3.

RNFL: the retinal nerve fiber layer is segmented by applying the k-means algorithm on the top part of the aligned image, around the ILM membrane, with $k=3$ classes. The aligned image has been denoised beforehand by the non linear diffusion filter. As the contrast is very high, the classification results are very homogeneous (Fig. 8) and the MRF classification is not required. The clusters corresponding to the higher mean intensity and connected to the ILM membrane are selected. From this first result, it is easy to deduce the contours of the RNFL, on both sides of the foveola, and to regularize them by applying the snake algorithm.

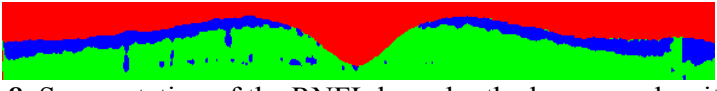

Fig. 8. Segmentation of the RNFL layer by the k-means algorithm.

OPL/ONL: the k-means/MRF segmentation process is applied on the foveal region (Fig. 9), defined horizontally by the clivus, limited vertically by the ILM or the RNFL and the ONL/IS boundary, with $k=3$ and $\beta=2$. The ONL appears as the cluster corresponding to the lowest mean intensity (label 1) just above the ONL/IS boundary. So the OPL/ONL boundary can be easily deduced from the labeled image. It is then regularized using the active contour algorithm (Fig. 9).
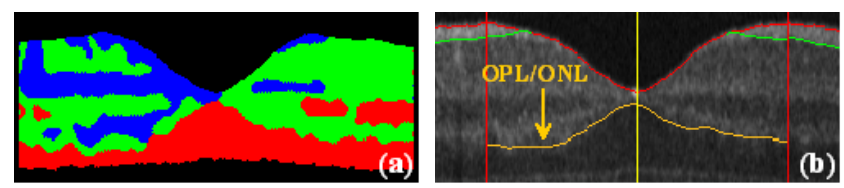

Fig. 9. Segmentation of the ONL layer: (a) k-means/MRF segmentation outputs (label 1 in red), (b) OPL/ONL localization.

GCL+IPL/INL: the region of interest is now more restricted, knowing the location of the OPL/ONL interface (Fig. 10). The method is applied with $k=2$ classes and $\beta=5$, on the left and right parts independently, since the average contrast may be slightly different on both sides. Clusters of low mean intensity (label 1) and connected to the upper boundary of the region of interest are fused with the surrounding cluster (label 2). The location of the searched boundary is then estimated at 4 y-coordinates $\left(y_{C l}, y_{C l} / 2\right.$, $\left.y_{C r} / 2, y_{C r}\right)$ and forced at the foveola $y_{F}$ to pass between the ILM and the OPL/ONL interface. These 5 points are linearly interconnected to provide a first estimate of the searched boundary (Fig. 10b). Finally, the labeled image is explored column by column from top to down. Pixels corresponding to a transition between label 2 and label 1 and close to the first estimate are marked as boundary points. These points serve as initialization of a snake, for the purpose of filling in the gaps and regularizing the curve (Fig.11).

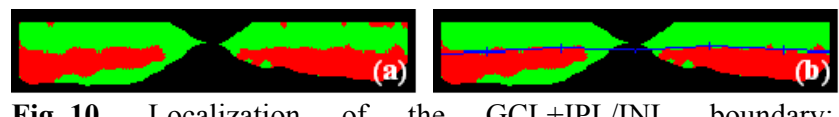

Fig. 10. Localization of the GCL+IPL/INL boundary: (a) k-means/MRF segmentation outputs (label 1 in red, label 2 in green), (b) first estimate of the boundary GCL+IPL/INL.

INL/OPL: a similar segmentation process is applied on the region limited by the two curves found previously.

\section{EXPERIMENTS AND RESULTS}

OCT imaging was performed on 72 images, from 25 normal subjects (either both eyes or only the right one was observed). A vertical and a horizontal cross-section were generally acquired for each eye. All subjects had a best corrected visual acuity of at least 80 points on the ETDRS (Early Trial Diabetic Retinopathy Study) charts, and a normal fundus. This study was conducted in accordance with French ethics regulation and all subjects gave informed consent to participate.

The automated detection of all layer interfaces was performed successfully on the 72 images, using the methods described in this paper. The mean processing time is around 2 minutes for each image (Matlab program running on a $2.4 \mathrm{GHz}$ Intel Core 2 DUO CPU). Only less than $1 \%$ of the interfaces were not accurately localized. This occurs for the detection of the ONL/IS or the 
OS/RPE boundary in some especially blurred images. Additionally to a visual assessment by an expert, a quantitative evaluation was performed on 14 images. These images have been chosen so as to be representative of the different quality and noise levels that occur in the complete set of images. They have been segmented manually by 5 different experts, using a graphical user interface (GUI) developed with Matlab. The evaluation then consists in comparing measures (Fig. 11, Table 1) obtained from the manual segmentations and from the automated ones.

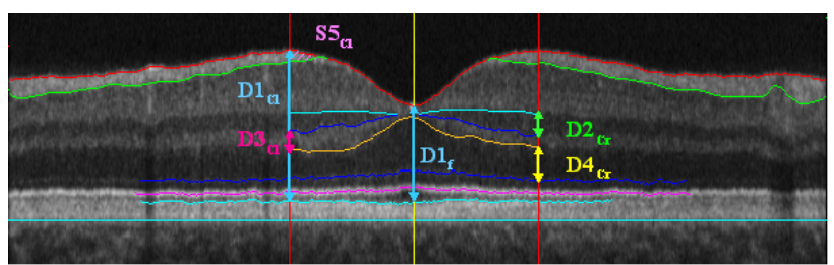

Fig. 11. Segmentation of the retinal layers and derived measures.

\begin{tabular}{|c|c|c|c|}
\hline Measures & $\begin{array}{c}\text { Automated (14) } \\
(\mu \mathrm{m})\end{array}$ & $\begin{array}{c}\text { Manual (14) } \\
(\mu \mathrm{m})\end{array}$ & $\begin{array}{c}\text { Automated (72) } \\
(\mu \mathrm{m})\end{array}$ \\
\hline $\mathbf{D 1}_{\mathbf{c l}}$ & $289.9 \pm 12.5$ & $287.3 \pm 11.8$ & $291.1 \pm 11.8$ \\
$\mathbf{D 1}_{\mathbf{f}}$ & $161.4 \pm 10$ & $166 \pm 9.3$ & $165.6 \pm 13.4$ \\
$\mathbf{D 1}_{\mathbf{c r}}$ & $274.8 \pm 11.8$ & $275.4 \pm 12.3$ & $279.8 \pm 14.3$ \\
$\mathbf{D 2}_{\mathrm{cl}}$ & $38.0 \pm 5.3$ & $42 \pm 5.3$ & $38.5 \pm 4.9$ \\
$\mathbf{D 2}_{\mathrm{cr}}$ & $38.4 \pm 5.5$ & $40.1 \pm 6.4$ & $38.3 \pm 5.3$ \\
$\mathbf{D 3}_{\mathrm{cl}}$ & $27.9 \pm 9.2$ & $25.6 \pm 9.6$ & $29.3 \pm 10.5$ \\
$\mathbf{D 3}_{\mathrm{cr}}$ & $27.1 \pm 8.9$ & $25.8 \pm 9.8$ & $26.2 \pm 10.5$ \\
$\mathbf{D 4}_{\mathrm{cl}}$ & $60.4 \pm 11.4$ & $66.7 \pm 11.8$ & $61.4 \pm 10.8$ \\
$\mathbf{D 4}_{\mathbf{f}}$ & $91.6 \pm 7.5$ & $92.9 \pm 7.9$ & $93.2 \pm 9.1$ \\
$\mathbf{D 4}_{\mathrm{cr}}$ & $66.6 \pm 11.6$ & $67.2 \pm 13.9$ & $62.2 \pm 14.7$ \\
$\mathbf{S 5}_{\mathrm{cl}}$ & $4125 \pm 1838$ & $6129 \pm 2767$ & $4924 \pm 2127$ \\
\hline
\end{tabular}

Table 1. Some measures obtained from the manual and automated segmentations: maximal $\left(D 1_{c l}, D 1_{c r}\right)$, foveal $\left(D 1_{f}\right)$, INL $(D 2)$, OPL (D3) and ONL (D4) thicknesses, RNLF surface (S5).

Table 1 indicates a very good agreement between the measures performed manually by the experts and those obtained from the automatic segmentation. Interestingly, computing the measures on the whole database provides similar mean values and slightly higher standard deviations (which was expected), which illustrates the representativeness of the chosen examples for the quantitative evaluation.

Finally, a distance between the manually traced curves and the ones obtained by the proposed method was calculated. The distance varies from 1.8 pixels for the RNFL/GCL+IPL interface, to 4 pixels for the OPL/ONL, which is again very accurate.

The proposed method was a basis for a preliminary study of variation of the morphology of foveal and perifoveal layers within a population of healthy subjects. We found that the profile of the fovea changed with axial length, with a relative increase of central ONL thickness which underlies a corresponding increase of foveal thickness. Independently of axial length, a large variability of the OPL/ONL complex was noted. On the opposite, the maximal retinal thickness was stable over a large range of axial length and had a low coefficient of variation. No gender-related differences were noted.

\section{CONCLUSION}

In this paper, a new automatic method for segmenting eight layers in OCT retinal images has been proposed. A quantitative evaluation has been performed on 14 images covering the usually observed variability, by comparing the results with those obtained manually by five experts. The quantitative results show a very good agreement and high correlation between the two sets of measures. Also the distance between the manually segmented interfaces and the automated ones shows the accuracy of our segmentation method, including for interfaces that were not addressed before in the literature, such as the inner and outer segments of the photoreceptors. Moreover a database of 72 images has been successfully segmented. A more detailed evaluation will be performed in future work.

\section{ACKNOWLEDGMENTS}

The authors would like to thank the medical team of the Clinical Investigation Center of the Quinze-Vingts hospital for contributing to the manual segmentations. This work was partially funded by a CIFRE grant from FOVEA and ANRT.

\section{REFERENCES}

[1] Topcon, "Topcon Europe medical BV," Dec. 2008; http://www.topcon-medical.eu/index.html .

[2] H. Ishikawa, D. M. Stein, G. Wollstein, S. Beaton, J. G. Fujimoto and J. S. Schuman, "Macular Segmentation with Optical Coherence Tomography," Invest. Ophthalmol. Visual Sci., vol. 46, pp. 2012-2017, 2005.

[3] D. Koozekanani, K. Boyer and C. Roberts, "Retinal Thickness Measurements from Optical Coherence Tomography Using a Markov Boundary Model," IEEE transaction on medical imaging, Vol. 20, No. 9, pp. 900-916, Sept. 2001.

[4] M. Baroni, P. Fortunat and A. La Torre, "Towards Quantitative Analysis of Retinal Features in Optical Coherence Tomography," Med. Eng. \& Phys., Vol. 29, No. 4, pp. 432-441, 2007.

[5] A. M. Bagci, M. Shahidi, R. Ansari, M. Blair, N. P. Blair and R. Zelkha, "Thickness Profile of Retinal Layers by Optical Coherence Tomography Image Segmentation," American Journal of Ophthalmology, Vol. 146 , No. 5 , pp. 679 - 687, Aug. 2008.

[6] D.C. Fernandez, H. M. Salinas and C. A. Puliafito, "Automated Detection of Retinal Layer Structures on Optical Coherence Tomography images," Optics Express, Vol. 13, No. 25, pp. 1020010216, Dec. 2005.

[7] M.K. Garvin, M.D. Abramoff, R. Kardon, S.R. Russell, W. Xiaodong and M. Sonka, "Intraretinal Layer Segmentation of Macular Optical Coherence Tomography Images Using Optimal 3-D Graph Search," IEEE Transaction on Medical Imaging, Vol. 27, No. 10, pp. 1495-1505, Oct. 2008.

[8] J. Weickert, B.M.T.H. Romeny and M.A. Viergever, "Efficient and Reliable Schemes for Nonlinear Diffusion Filtering," IEEE Trans. on Image Processing, Vol. 7, No. 3, pp. 398 - 410, 1998.

[9] C. Xu and J. L. Prince, "Snakes, Shapes, and Gradient Vector Flow," IEEE Trans. on Image Processing, Vol. 7, No. 3, pp. 359369, Mar. 1998.

[10] R.G Brown and P.Y.C Hwang, "The Discrete Kalman Filter, State-Space Modeling and Simulation," in Introduction to Random Signals and Applied Kalman Filtering, 3th Ed. New York: J.W, 1997, Ch. 5, Sec. 5.5, pp. 214-220.

[11] S. Geman and D. Geman, "Stochastic Relaxation, Gibbs Distribution, and the Bayesian Restauration of Images," IEEE Transactions on Pattern Analysis and Machine Intelligence, Vol. PAMI-6, No. 6, pp. 721-741, Nov. 1984. 